\section{Severe lower limb ischemia by massive arterial thrombosis revealing an acute myeloid leukemia needing for leg amputation: clinical and emotional aspects related to the communication with the patient and his family}

\author{
Paolo D'Angelo, ${ }^{1}$ Calogero Taormina, ${ }^{1}$ \\ Clara Mosa, ${ }^{1}$ Floriana Di Marco, ${ }^{1}$ \\ Fabrizio Valentino,2 Angela Trizzino, 1 \\ Paola Guadagna, ${ }^{1}$ Francesco Talarico ${ }^{2}$ \\ 1Pediatric Hematology and Oncology \\ Department, and 2Vascular Surgery \\ Department, A.R.N.A.S Civico, Di Cristina \\ e Benfratelli Hospitals, Palermo, Italy
}

\section{Abstract}

Large vessel thrombosis is a very rare clinical presentation of acute leukemia, generally associated with coagulopathy, usually characteristic of acute promyelocytic leukemia. A 13year-old boy with a previously undiagnosed acute myeloid leukemia was referred to our hospital with acute ischemia of the right lower limb due to occlusion of the right external iliac artery, treated with emergency double surgical thromboembolectomy and chemotherapy. The thrombotic complication resulted in leg amputation. Now the boy is well in complete remission, with a good social integration and quality of life, 30 months after completing treatment. The report highlights the crucial role of early diagnosis and subsequent chemotherapy in avoiding amputation. We particularly focused critical and emotional aspects related to the communication about the leg amputation with the patient and his family.

\section{Introduction}

Thromboembolism is a well-known complication of acute myeloid leukemia (AML) especially in patients with high white blood cell count, presence of FLT3-ITD and expression of CD2. ${ }^{1}$ However, spontaneous vaso-occlusion in the main arteries is very rare, especially in patients with no pre-existing vasculopathy. In patients with acute leukemia, the risk of thrombosis is not negligible. Thrombosis can be a presenting symptom at diagnosis in a significant proportion of cases with acute promyelocytic leukemia (APL) (9.6\%) and other AML (3.2\%); a similar rate of thrombosis can occur during the subsequent course of the disease. The incidence of symptomatic thrombosis at diagnosis is relatively low in acute lymphoblastic leukemia (ALL) patients (1.4\%), but increases significantly by further treatment up to $10.6 \% .^{2}$ AML can be associated with hemorrhagic complications subsequent to disseminated intravascular coagulation (DIC), most of all in APL, which is more frequently related to thromboembolic occlusions of arterial large vessels. ${ }^{3}$ We report a case of severe recurrent acute arterial thromboembolism at the onset of an acute myeloid leukemia FAB M1, which finally required a leg amputation. Emotional aspects are analyzed in detail.

\section{Case Report}

A 13-year-old boy suffering from fever for 6 days, as well as pain and paresthesia at the right feet, was admitted to our Emergency Unit and immediately underwent angiographic computed tomography (CT) that showed the occlusion of the right external iliac artery (Figure 1A). The boy underwent immediate surgical thromboembolectomy of the right iliac and femural arteries immediately, and subsequently continuous infusion of heparin and urokinase.

Due to blood examination results that revealed anemia ( $\mathrm{Hb} 8.3 \mathrm{~g} / \mathrm{dL}$ ), leukocytosis (white blood cells were $92,900 / \mathrm{mm}^{3}$ ) and mild thrombocytopenia (platelets were 118,000/ $\mathrm{mm}^{3}$ ), the patient was transferred to our department. Morphology and flow cytometry of the peripheral blood defined a diagnosis of FAB M1 AML. On physical examination the boy had severe hypothermia from the middle third of the right leg, with no detectable pulse and diffuse marbled skin, especially in the medial region. No other clinical signs or symptoms were observed. Echocolordoppler of chest and abdomen were normal. Other laboratory examinations revealed LDH 910 IU/L, AST/ALT 17/12 IU/L, PT 14", INR 1.29, fibrinogen $246 \mathrm{mg} / \mathrm{dL}$, aPTT 29.10", C-protein 31\% (normal values 70$140 \%$ ), while the study of lupus anticoagulant, MTHFR and Leyden $V$ factor were normal.

The boy underwent supportive treatment immediately including hyper-hydration (glucose and sodium chloride solution 3000 $\mathrm{mL} / \mathrm{sqm} /$ day $)$ with bicarbonate $(100$ $\mathrm{mmol} / \mathrm{sqm})$, allopurinol $(15 \mathrm{mg} / \mathrm{kg} /$ day in 3 doses) and continuous infusion of heparin (20,000 IU/day) and prostaglandin (1 $\mathrm{ng} / \mathrm{kg} / \mathrm{min})$. Bone marrow examination showed a complete metaplasia (atypical myeloblasts $90 \%$ ) confirming the diagnosis of FAB M1 AML (Figure 1B). On the second day, the clinical and imaging conditions persisted, so a second thromboembolectomy of the anterior and posterior tibial arteries and of the
Correspondence: Paolo D’Angelo, Unità Operativa di Oncoematologia Pediatrica, A.R.N.A.S. Civico, Di Cristina e Benfratelli, Piazza Nicola Leotta, 4, 90127 Palermo, Italy. Tel.: +39.91.6664142 - Fax: +39.91.666412 E-mail: oncoematoped@arnascivico.it

Key words: Large vessels arterial thrombosis; Acute myeloid leukemia; Chemotherapy; Leg amputation; Communication emotional aspects.

Acknowledgements: the authors wish to thank Prof. Frank Adamo for revising the manuscript and the parents' Association A.S.L.T.I. - Liberi di crescere Onlus for the financial support.

Contributions: PD, CT and CM, manuscript writing and references searching; PD, CT, and AT manuscript reviewing; PD, CT, PG, AT, FV and FT were equally involved in the clinical management of the patient.

Conflict of interest: the authors declare no conflict of interest.

Received for publication: 11 September 2016.

Revision received: 2 October 2016.

Accepted for publication: 8 October 2016 .

This work is licensed under a Creative Commons Attribution NonCommercial 4.0 License (CC BYNC 4.0).

CC Copyright P. D'Angelo et al., 2016

Licensee PAGEPress, Italy

Pediatric Reports 2016; 8:6885

doi:10.4081/pr.2016.6885

pedidial artery with local infusion of heparin and locoregional thrombolysis with urokinase was performed. The vascular tree appeared diffusely stuffed by abundant lard-like material consisting of recently formed neoplastic tissue. After this second procedure physical examination of the feet was consistent with a possible recovery of the vascular damage (Figure 2A,B); chemotherapy with intravenous continuous infusion of cytosine arabinoside at the dose of $200 \mathrm{mg} / \mathrm{sqm} / \mathrm{day}$, with the aim of decreasing the blast count, was started immediately after the second thromboembolectomy. Four days later, the ischemic and dystrophic progression of the inferior third of the leg and feet, confirmed irreversible damage, with a hypothermic level raised to the superior third of the leg and a significant increase of muscular necrosis (Figure 2C,D). Also the laboratory parameters (LDH 3305 IU/L, CPK 10,084 IU/L, myoglobin $1541 \mathrm{ng} / \mathrm{mL}$ ), determined the need for an above-knee leg amputation. Sodium heparin treatment was continued during the entire hospitalization. Two weeks after amputation the boy underwent the first course of chemotherapy according to our National 
Protocol AIEOP LMA 2002/1, with the ICE schedule (idarubicin $10 \mathrm{mg} / \mathrm{sqm} /$ day for 3 days, cytosine arabinoside $200 \mathrm{mg} / \mathrm{sqm} /$ day for 7 days and etoposide $100 \mathrm{mg} / \mathrm{sqm} /$ day for 5 days). A complete remission was documented after the first course; so the scheduled treatment (a $2^{\text {nd }}$ ICE course and three more consolidation courses), ${ }^{4}$ was completed without problems or significant side effects and now the boy is well and in first complete remission, with a very good quality of life, 30 months after the discontinuation of treatment.

\section{Discussion}

Thromboembolism is a well-known complication of some cancers including AML. However, spontaneous vaso-occlusion in the main arteries is very rare, especially in children. AML may be more frequently associated with coagulopathy, predominantly severe bleeding diathesis caused by DIC and/or hyperfibrinolysis. Hemostasis disturbances are most of all characteristic of acute promyelocytic leukemia (AB M3). However, thromboembolic events such as arterial occlusion localized to the large vessels at presentation are very rare and almost exclusively linked to APL.5-8

In our patient acute arterial thromboembolism was the only initial symptom at presentation in AML FAB M1, starting 6 days before the admission with pain and paresthesia at the right feet. The ischemic events in our patient most likely resulted from leukemia as the thrombus material included many leukemic blasts. The unblocking of the iliac artery resulted in a prompt reperfusion, but intraarterial administration of fibrinolytic agent was probably ineffective in the other arteries, because of the particular nature of the thrombus, almost entirely composed by agglutinated blasts, that prevented the reperfusion of the microcirculation. The thrombotic complications resulted in leg amputation, because the patient was probably referred too late (pain and paresthesia at right feet and fever since 6 days); an earlier diagnosis and a prompt start of chemotherapy could have probably avoided this dramatic surgery.

Despite imminent leg amputation and extremely high infectious risk, chemotherapy was continued just till to a couple of hours before, and subsequently, 15 days after leg amputation, treatment was restarting according to the protocol, without any dose modification. The clinical course of cessation of the ischemic events and a fast reduction of the blasts in the peripheral blood after chemotherapeutic treatment highlights the important, life-saving role of early chemotherapy even under adverse circumstances, that probably avoided further vascular complication.
One of the most important and critical problems in this clinical situation was surely the management of communication. The stress and discomfort were managed by the entire care group (physicians, nurses and psychologists).

The therapeutic relationship established from the beginning of the patient's care was crucial. Communication proved to be only the beginning of long-term assistance of the patient and his family, including the redefining the patient's perception of his own body and future plans. Our team shared the emotional burden and required all members to deal with this event. When the parents agreed to the planned therapeutic procedure, we informed the patient in order to prepare him for the surgery. While communicating the doctor was supported by the presence of a psychologist and nurses. One meeting was not sufficient for communication, but in the subsequent period the patient had many other chances to meet with the medical staff.

As already described in the literature, $, 9,10$ the psychological support activities can be pursued on two levels of intervention: medical psychology support, involving the whole team and clinical psychological participation by a psychotherapist inserted into the care group.

The relationship with the doctors and nurs-
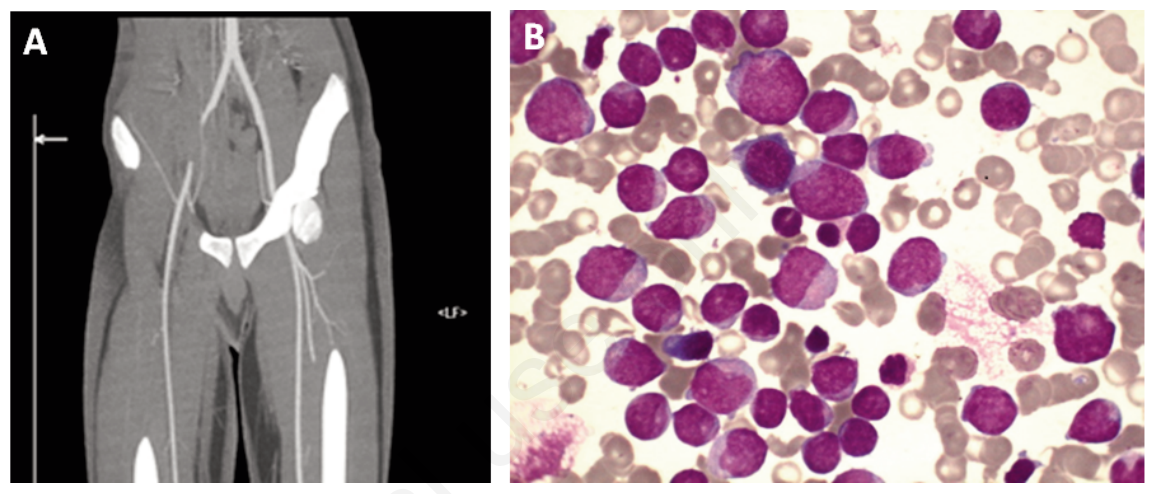

Figure 1. A) Angiographic computed tomography showed the complete occlusion of the right external iliac artery. B) Bone marrow examination showed a complete metaplasia (atypical myeloblasts $\mathbf{9 0 \%}$ ) confirming the diagnosis of acute myeloid leukemia FAB M1.
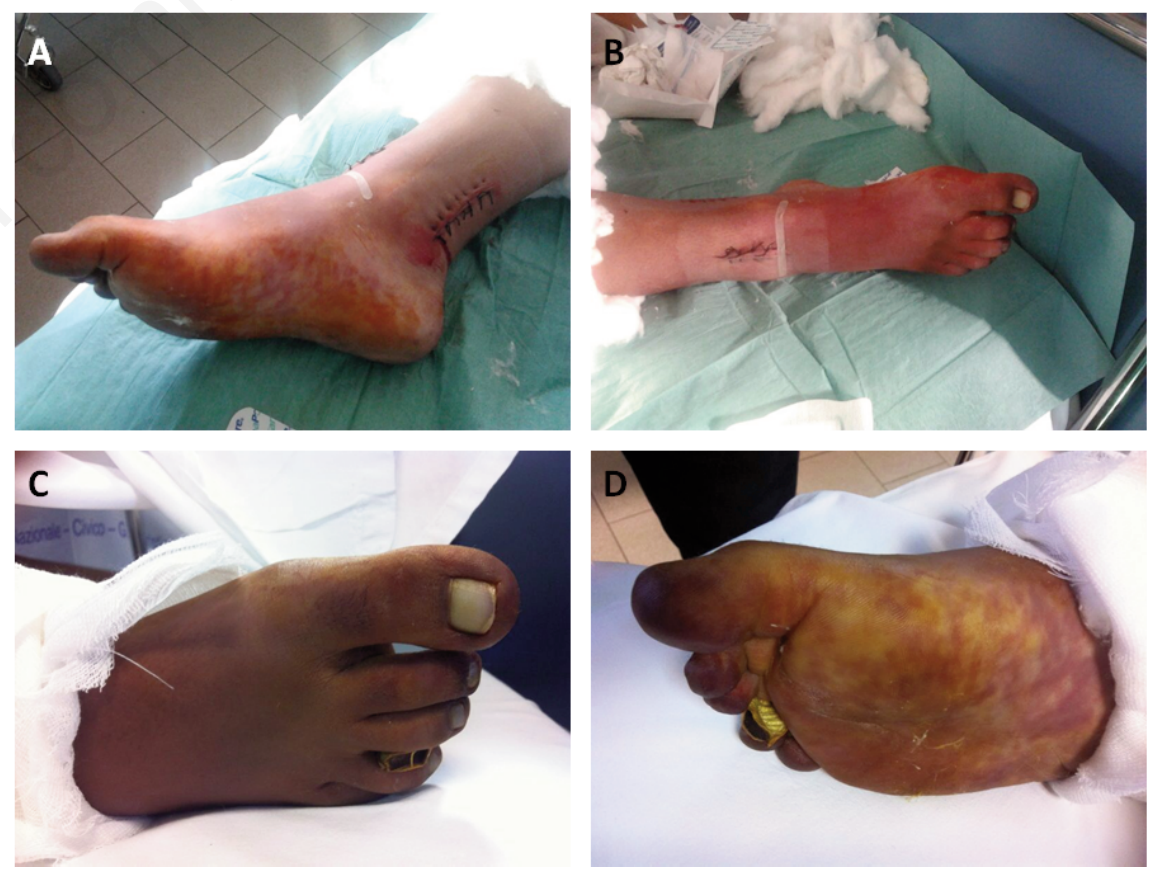

Figure 2. A,B) after the second thromboembolectomy clinical status of the feet suggested a possible recovery of the vascular status and a reversible damage. $\mathrm{C}$ ) the ischemic and dystrophic progression of the feet confirmed an irreversible damage. D) the feet showed an evident color modification with diffuse marbled skin, especially in the medial region. 
es was extremely important; both medical and nursing staff could provide valuable input on their responsibility for the patient, establishing a relationship based on deep trust, ensuring the fundamental experience of being held, be protected and be guided even in such adverse circumstances.9,10 The family's feelings about our take-charge approach, and the sharing of emotional moods, also made it possible to prevent the chronic stress due to the management of adverse events. ${ }^{11}$

Clinical psychological intervention took place through clinical interviews and supportive psychotherapy sessions. It was possible to work on all emotional aspects of amputation (fear, anxiety), future plans, the redefinition of the patient's own body image, perceptions related to phantom limb syndrome, and the chance to show himself (relational and social aspects) in the new condition.

The work with the parents was important as specific parenting skills support, useful to help their young son with such a difficult adjustment, and avoid any negative outcome in the adaptation and accommodation of the event. 12

\section{Conclusions}

Large vessel thrombosis is a very rare clinical presentation of AML. Our report describe a boy with acute ischemia of the right lower limb due to occlusion of the right external iliac artery, as the only clinical symptom of AML, treated with prompt double surgical thromboembolectomy, chemotherapy and subsequent leg amputation. Since the boy is well in continue complete remission, with a good social integration and quality of life, the report highlights the important life-saving role of early and full chemotherapy, even if an earlier recognition and therapeutic intervention could have prevented the amputation of the leg, and the relevance of an adequate management of critical and emotional aspects related to the communication about the leg amputation both with the patient and his family.

\section{References}

1. Breccia M, Avvisati G, Latagliata R, et al. Occurrence of thrombotic events in acute promyelocytic leukemia correlates with consistent immunophenotypic and molecular features. Leukemia 2007;21:79-83.

2. De Stefano V, Sorà F, Rossi E, et al. The risk of thrombosis in patients with acute leukemia: occurrence of thrombosis at diagnosis and during treatment. J Thromb Haemost 2005;3:1985-92.

3. Rashidi A, Silverberg ML, Conkling PR, et al. Thrombosis in acute promyelocytic leukemia. Thromb Res 2013;131:281-9.

4. Pession A, Masetti R, Rizzari C, et al. Results of the AIEOP AML 2002/01 multi- center prospective trial for the treatment of children with acute myeloid leukemia. Blood 2013;122:170-8.

5. Rolston DD, Rubin S, Topolsky D, et al. Arterial occlusions as a presenting feature of acute promyelocytic leukemia. Am J Clin Oncol 1998;21:436-7.

6. Di Giovanni RJ Jr, Crilley P, Kerstein MD. Peripheral arterial occlusion in acute promyelocytic leukemia. Cardiovasc Surg 1999;7:58-60.

7. Kalk E, Goede A, Rose P. Acute arterial thrombosis in acute promyelocytic leukaemia. Clin Lab Haematol 2003;25: 267-70.

8. Trottier-Tellier F, Durand M, Kolan C, et al. Recurrent Arterial and Venous Thromboemboli as Initial Presentation of Acute Promyelocytic Leukemia. J Clin Med Res 2014;6:388-91.

9. Clerici CA, Ferrari A, Massimino M, et al. Aspetti psicologici dei pazienti amputati in età pediatrica per malattie neoplastiche. Ital J Pediatr 2001;27:887-92.

10. Ferrari A, Clerici CA, Spreafico F, et al. Psychological support in children and adolescents with cancer when amputation is required. Med Pediatr Oncol 2002;38:2615

11. Rispoli L. Esperienze di base è sviluppo del Sé. Milan: FrancoAngeli; 2004.

12. Rispoli L, Di Nuovo S, Genta E. Misurare lo stress. Milan: FrancoAngeli; 2000. 